\title{
Phytoprotection
}

phytoprotection

\section{Liste des arbitres 2004-2006}

List of Reviewers 2004-2006

Volume 88, numéro 1, avril 2007

URI : https://id.erudit.org/iderudit/016400ar

DOI : https://doi.org/10.7202/016400ar

Aller au sommaire du numéro

Éditeur(s)

Société de protection des plantes du Québec (SPPQ)

ISSN

0031-9511 (imprimé)

1710-1603 (numérique)

Découvrir la revue

Citer ce document

(2007). Liste des arbitres 2004-2006. Phytoprotection, 88(1), 31-33.

https://doi.org/10.7202/016400ar d'utilisation que vous pouvez consulter en ligne.

https://apropos.erudit.org/fr/usagers/politique-dutilisation/ 


\section{Liste des arbitres 2004-2006 \\ List of Reviewers 2004-2006}

Le comité de rédaction de PHYTOPROTECTION veut reconnaître l'excellente contribution de nombreux collaborateurs qui ont généreusement participé au processus d'évaluation des manuscrits. Qu'ils trouvent ici l'expression de nos remerciements les plus sincères.

The Editorial Board of PHYTOPROTECTION would like to acknowledge the excellent contribution of many collaborators who generously participated in the evaluation of manuscripts. May they find here the expression of our most sincere gratitude.

Anagnostakis, S.L. Connecticut Agricultural Experiment Station, New Haven, Connecticut

Akhtar, Y.

University of British Columbia, Vancouver, British Columbia

Anderson, J .

Purdue University, Lafayette, Indiana

Asselin, A.

Université Laval, Québec, Québec

Avis, T.

Université Laval, Québec, Québec

Barasubiye, $\mathrm{T}$.

Agriculture and Agri-Food Canada, Ottawa, Ontario

Beaulieu, C.

Université de Sherbrooke, Sherbrooke, Québec

Bélair, G.

Agriculture et Agroalimentaire Canada, Saint-J ean-sur-Richelieu, Québec

Bélanger, R.

Université Laval, Québec, Québec

Berger, R.

University of Florida, Gainesville, Florida

Bernier-Cardou, M.

Service canadien des forêts, Québec, Québec

Berthiaume, R.

Service canadien des forêts, Québec, Québec

Bigras, $\mathrm{F}$.

Service canadien des forêts, Québec, Québec

Boiteau, G.

Agriculture et Agroalimentaire Canada, Fredericton, Nouveau-Brunswick

Boland, G.J .

University of Guelph, Guelph, Ontario

Brodo, F.

Self-employed researcher, Ottawa, Ontario

Bromfield, E.

Agriculture and Agri-Food Canada, Ottawa, Ontario

Chain, $F$.

Université Laval, Québec, Québec

Charest, P.-M .

Université Laval, Québec, Québec

Cilas, C.

CIRAD, Montpellier, France

Couture, L.

Agriculture et Agroalimentaire Canada, Québec, Québec

DiTommaso, A.

Cornell University, Ithaca, New York

Doohan, D.

Ohio State University, Wooster, Ohio

Dostaler, D.

Université Laval, Québec, Québec

Felix, J.

Ohio State University, Wooster, Ohio 
Fitzpatrick, S.

Fréchette, $B$.

Gossen, B.

Goulet, $\mathrm{H}$.

Gulya, T.

Hager, A.

Harrison, K.

Hogue, R.

Hwang, S.-F.

Hynes, R.K.

Isman, M.

J ensen, $\mathrm{K}$.

J udd, G.

Kegode, G.

Kurle, J .

Laflamme, G.

Lalande, R.

Lamontagne, M.

Langevin, F.

Li, S.

Martin, J.

Martinez, C.

MacDonald, W.

Maund, C.

McCallum, B.

McSorley, R.

Michaud, D.

Mpofu, S.

Navi, S.S.

Nolte, P.

O'Hara, J .

Ouellette, G.B.

Ploeg, A.

Pouleur, S.

Rashid, K.Y.
Agriculture and Agri-Food Canada, Agassiz, British Columbia

Université du Québec à Montréal, Montréal, Québec

Agriculture and Agri-Food Canada, Saskatoon, Saskatchewan

Agriculture and Agri-food Canada, Ottawa, Ontario

USDA- ARS, Fargo, North Dakota

University of Illinois, Urbana, Illinois

Ohio State University, Columbus, Ohio

Institut de recherche et de développement en agroenvironnement, Québec, Québec

Alberta Research Council, Vegreville, Alberta

Agriculture and Agri-Food Canada, Saskatoon, Saskatchewan

University of British Columbia, Vancouver, British Columbia

Agriculture and Agri-Food Canada, Kentsville, Nova Scotia

Agriculture and Agri-Food Canada, Summerland, British Columbia

North Dakota State University, Fargo, North Dakota

University of Minnesota, St. Paul, Minnesota

Service canadien des forêts, Québec, Québec

Agriculture et Agroalimentaire Canada, Québec, Québec

Université de Moncton, Edmunston, Nouveau-Brunswick

Agriculture et Agroalimentaire Canada, Québec, Québec

Canadian Forest Service, Ottawa, Ontario

University of Kentucky, Princeton, Kentucky

Premier Tech, Rivière-du-Loup, Québec

West Virginia University, Morgantown, West Virginia

New Brunswick Department of Agriculture, Fredericton, New Brunswick

Agriculture and Agri-food Canada, Winnipeg, Manitoba

University of Florida, Gainesville, Florida

Université Laval, Québec, Québec

Olds College, Olds, Alberta

lowa State University, Ames, lowa

University of Idaho, Moscow, Idaho

Agriculture and Agri-Food Canada, Ottawa, Ontario

Service canadien des forêts, Québec, Québec

University of California, Riverside, California

Agriculture et Agroalimentaire Canada, Québec, Québec

Agriculture and Agri-Food Canada, Morden, Manitoba 
Reddy, K.

Rioux, D.

Rioux, S.

Rowe, D.E.

Simon, U.K.

Schutte, B.L.

Subramanyam, S.

Timper, P.

Toussaint, V.

Trudel, R.

Tweddell, R.

Wang, K-H.

Zhou, J .
USDA-ARS, Stoneville, Mississippi

Service canadien des forêts, Québec, Québec

Centre de recherche sur les grains, Québec, Québec

USDA-ARS, Mississippi State, Mississippi

Université de Tübingen, Tübingen, Allemagne

Ohio State University, Colombus, Ohio

Purdue University, Lafayette, Indiana

USDA-ARS, Tifton, Georgia

Agriculture et Agroalimentaire Canada, Saint-J ean-sur-Richelieu, Québec

Société de protection des forêts contre les insectes et maladies, Québec, Québec

Université Laval, Québec, Québec

University of Florida, Gainesville, Florida

North Dakota State University, Fargo, North Dakota 